\title{
FABRICATION AND CHARACTERIZATION OF GaN JUNCTIONFIELD EFFECT TRANSISTORS
}

\author{
L. Zhang, ${ }^{*}$ L. F. Lester,** A. G. Baca, ${ }^{*}$ R. J. Shul, ${ }^{*}$ P. C. Chang, ${ }^{*}$ C. G. Willison,* \\ U. K. Mishra, ${ }^{* * *}$ S. P. Denbaars, ${ }^{* * *}$ and J. C. Zolper**** \\ * Sandia National Laboratories, Albuquerque, NM 87185 \\ ** University of New Mexico, Albuquerque NM 87106 \\ *** University of California, Santa Barbara, CA 93106 \\ ****Office of Naval Research, Arlington, VA 22217
}

\begin{abstract}
Junction field effect transistors (JFET) were fabricated on a GaN epitaxial structure grown by metal organic chemical vapor deposition. The DC and microwave characteristics, as well as the high temperature performance of the devices were studied. These devices exhibited excellent pinch-off and a breakdown voltage that agreed with theoretical predictions. An extrinsic transconductance $\left(g_{m}\right)$ of $48 \mathrm{mS} / \mathrm{mm}$ was obtained with a maximum drain current $\left(I_{D}\right)$ of $270 \mathrm{~mA} / \mathrm{mm}$. The microwave measurement showed an $f_{T}$ of $6 \mathrm{GHz}$ and an $f_{\max }$ of $12 \mathrm{GHz}$. Both the $I_{D}$ and the $g_{m}$ were found to decrease with increasing temperature, possibly due to lower electron mobility at elevated temperatures. These JFETs exhibited a significant current reduction after a high drain bias was applied, which was attributed to a partially depleted channel caused by trapped electrons in the semi-insulating GaN buffer layer.
\end{abstract}

\section{INTRODUCTION}

Wide bandgap $\mathrm{GaN}$ and its related materials have great potential for high power and high temperature microwave electronic device applications owing to their low intrinsic carrier concentration, high breakdown field, high saturation velocity, and excellent chemical stability [1-3]. A significant amount of effort has been devoted to the development of various GaN-based field effect transistors (FETs) [4-8], which has led to the demonstration of high performance GaN/AIGaN MODFETs [9-12]. Compared to MESFETs and MODFETs, JFETs provide a higher gate voltage swing and a lower reverse gate leakage current due to a higher built-in potential of the p-n junction gate than the Schottky gate used in MESFETs and MODFETs. This is especially important for high temperature operation. In addition, the junction gate is metallurgically more stable and environmentally more robust than a Schottky gate since it is effectively buried beneath the surface and is subjected to high temperature during crystal growth. Therefore, JFETs are expected to withstand higher temperature operation than MESFETs and MODFETs. In this paper, we report the fabrication and characterization of epitaxially grown GaN JFETs.

\section{MATERIAL AND FABRICATION}

The epitaxial structure of GaN JFETs was grown by metal organic chemical vapor deposition (MOCVD) on a C-plane sapphire substrate. The layer structure consisted of a $4.2 \mu \mathrm{m}$ semi-insulating GaN buffer layer, a $950 \AA$ Si-doped n-GaN channel, a $60 \AA$ undoped $\mathrm{GaN}$, and a $500 \AA \AA^{\circ} \mathrm{Mg}$-doped p-GaN. The sample was annealed at $850{ }^{\circ} \mathrm{C}$ for 15 
sec in $\mathrm{N}_{2}$ to activate the $\mathrm{Mg}$ dopant. Hall measurements showed a free carrier concentration of $1.3 \times 10^{18}, 2.4 \times 10^{18}$, and $6.1 \times 10^{14} \mathrm{~cm}^{-3}$ in the $\mathrm{p}-\mathrm{GaN}, \mathrm{n}-\mathrm{GaN}$, and SI-GaN, respectively. The electron mobility in the $\mathrm{n}-\mathrm{GaN}$ active layer was $270 \mathrm{~cm}^{2} / \mathrm{V}-\mathrm{sec}$. The fabrication process began with a mesa isolation etch in an inductively coupled $\mathrm{BCl}_{3} / \mathrm{Cl}_{2} / \mathrm{Ar}$ plasma. Next, a gate metal of $\mathrm{Ni} / \mathrm{Au} / \mathrm{Ni}$ was e-beam evaporated on top of the mesa and used as the mask for the self-aligned source-drain etch in a $\mathrm{BCl}_{3} / \mathrm{Cl}_{2} / \mathrm{Ar}$ ICP discharge. The device was completed with $\mathrm{Ti} / \mathrm{Al}$ source and drain ohmic contact metallization. Postmetallization annealing was not performed. A transmission line method (TLM) measurement showed an as-deposited source and drain ohmic contact resistance of $4.2 \Omega$ $\mathrm{mm}$, a specific contact resistance of $5 \times 10^{-5} \Omega-\mathrm{cm}^{2}$, and a sheet resistance of 4700 $\Omega /$ square, respectively. These values were relatively large, possibly due to plasma induced damage and an overetched source and drain region.

\section{RESULTS AND DISCUSSION}

Table I summarizes the DC and microwave result of a $0.8 \mu \mathrm{m}$ X $50 \mu \mathrm{m}$ GaN JFET with a source-drain spacing of $3 \mu \mathrm{m}$. A maximum $I_{D}$ of $270 \mathrm{~mA} / \mathrm{mm}$ and a maximum $g_{m}$ of $48 \mathrm{mS} / \mathrm{mm}$ were measured at $V_{G}=1 \mathrm{~V}$. A $R_{S}$ of $8.5 \Omega$-mm and a $R_{D}$ of $13 \Omega$-mm were obtained using the end resistance measurement technique. From the above result, an intrinsic transconductance $\left(g_{m 0}\right)$ of $81 \mathrm{mS} / \mathrm{mm}$ was calculated. The channel was completely pinched off at a threshold voltage of $V_{G}=-8 \mathrm{~V}$, with an $I_{D}=210 \mu \mathrm{A} / \mathrm{mm}$ at $V_{D}=15 \mathrm{~V}$. A gate-drain diode reverse breakdown voltage of $56 \mathrm{~V}$ was achieved, which corresponded to a breakdown field of $2.5 \times 10^{6} \mathrm{~V} / \mathrm{cm}$. The fact that the breakdown field approached the theoretical predicted breakdown field of GaN indicated that the relatively small breakdown voltage of the JFET as compared to the reported GaN MESFETs and MODFETs [4,12] was primarily due to the high doping concentration in the $n-G a N$. The gate leakage current of our JFETs was large as compared to GaN MODFETs [13,14], possibly due to the plasma induced damage to the junction $[15,16]$. The forward turn-on voltage of the gate diode was $\sim 1 \mathrm{~V}$ using a $1 \mathrm{~mA} / \mathrm{mm}$ current criterion. This value is only $30 \%$ of the bandgap energy of GaN. Pernot, et. al. have also reported a low turn-on voltage of $1.2 \mathrm{~V}$ on $\mathrm{GaN}$ p-n diodes [17]. The cause of this low turn-on is not known at present but may result from defect levels in the $\mathrm{GaN}$.

\begin{tabular}{|l|l|}
\hline maximum drain current $\left(I_{D}\right)$ & $270 \mathrm{~mA} / \mathrm{mm}$ \\
\hline threshold gate voltage $\left(V_{T}\right)$ & $-8 \mathrm{~V}$ \\
\hline knee voltage $\left(V_{\text {knee }}\right)$ & $8 \mathrm{~V}$ \\
\hline gate-drain diode breakdown voltage & $56 \mathrm{~V}$ \\
\hline gate turn-on voltage & $\sim 1 \mathrm{~V}$ \\
\hline extrinsic transconductance $\left(g_{m}\right)$ & $48 \mathrm{~ms} / \mathrm{mm}$ \\
\hline parasitic source resistance $\left(R_{S}\right)$ & $8.5 \Omega-\mathrm{mm}$ \\
\hline parasitic drain resistance $\left(R_{D}\right)$ & $13 \Omega-\mathrm{mm}$ \\
\hline cut-off frequency $\left(f_{T}\right)$ & $6 \mathrm{GHz}$ \\
\hline maximum frequency $\left(f_{\max }\right)$ & $12 \mathrm{GHz}$ \\
\hline
\end{tabular}

Table I: DC and microwave characteristics of a $0.8 \mu \mathrm{m}$ X $50 \mu \mathrm{m}$ GaN JFET. 


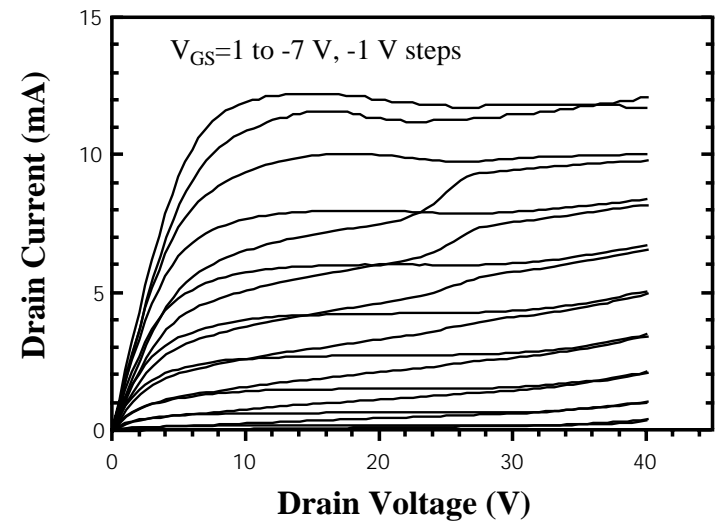

Figure 1: $I_{D^{-}} V_{D}$ characteristic showing the drain current collapse at $V_{D}<25 \mathrm{~V}$ due to a partially depleted channel by electrons trapped in the SI-GaN. The solid curves were measured individually with 5 min of illumination to release trapped electrons in the SIGaN. The dashed ones were measured in rapid recession.

Similar to what Binari and co-workers have reported on GaN MESFETs [4], a significant reduction in $\mathrm{I}_{\mathrm{D}}$ occurred in our JFETs after they were subjected to a high $V_{D}$. Figure 1 shows two sets of $I_{D^{-}} V_{D}$ curves measured up to $V_{D}=40 \mathrm{~V}$ under white-light illumination. The solid curves were measured individually with 5 minutes of illumination before each curve was taken while the dashed curves were measured in rapid succession. The upper dashed trace $\left(V_{G}=1 \mathrm{~V}\right)$ is nearly identical to the upper solid trace since they were both subjected to a long illumination. The subsequent dashed curves exhibited a large decrease in $I_{D}$ at $V_{D}<25 \mathrm{~V}$. This current collapse effect was not obvious unless $V_{D}$ was increased above $20 \mathrm{~V}$. The reduction in $I_{D}$ was caused by high-field injection and subsequent trapping of electrons in the SI-GaN, which depleted part of the active channel from the backside. We have demonstrated that these trapped electrons were located at the drain side of the channel where the electrical field was the highest [18]. At $V_{D}<5 \mathrm{~V}$, the transistor was below saturation and the current was limited by $R_{S}, R_{D}$, and the channel resistance, $R_{C H}$. Since the channel was partially depleted by the trapped electrons, a lower channel conductance was obtained for the dashed $I_{D^{-}} V_{D}$ curves as compared to the solid ones in Figure 1. Above the knee voltage, it was assumed that the channel consisted of a velocitysaturated section in parallel with a space-charge region caused by trapped electrons in the GaN buffer. The latter was evidenced by the nearly constant output conductance between the knee voltage and $V_{D}=25 \mathrm{~V}$. The rapid increase in $I_{D}$ of the collapsed curves at $V_{D} \sim 25 \mathrm{~V}$ may indicate a local breakdown of the space charge region. Therefore, regardless of illumination, $\mathrm{I}_{\mathrm{D}}$ at $V_{D}>25 \mathrm{~V}$ was limited by an undepleted, velocity-saturated region on the drain side of the space charge layer.

The microwave performance of the JFETs was characterized using an HP 8510 network analyzer. A unity current-gain cutoff frequency $\left(f_{T}\right)$ of $6 \mathrm{GHz}$ and a maximum frequency $\left(f_{\max }\right)$ of $12 \mathrm{GHz}$ were obtained at $V_{D}=15 \mathrm{~V}$ and $V_{G}=0 \mathrm{~V}$ from the small-signal $\mathrm{S}$-parameters. These values were comparable to the reported $f_{T}$ and $f_{\max }$ on $\mathrm{GaN}$

MESFETs and GaN/AIGaN MODFETs with a similar gate length $[19,20]$. The intrinsic $f_{T}$ of our JFETs would be higher by taking into account the large source and drain resistance. 
Following the derivation made by Tasker and Hughes [21], we were able to calculate $f_{T O}$ of the JFETs by de-embedding the effect of the parasitic $R_{S}$ and $R_{D}$ from measured $f_{T}$. From [21], the extrinsic cut-off frequency

$$
f_{T}=\frac{g_{m 0} / 2 \pi}{\left[C_{G S}+C_{G D}\right] \cdot\left[1+\left(R_{S}+R_{D}\right) / R_{D S}\right]+C_{G D} \cdot g_{m 0} \cdot\left(R_{s}+R_{D}\right)}
$$

where $C_{G S}, C_{G D}$, and $R_{D S}$ are the gate-source, gate-drain capacitance, and output resistance, respectively. Rearranging this equation, the intrinsic cut-off frequency

$$
f_{T 0} \equiv \frac{g_{m 0}}{2 \pi\left(C_{G S}+C_{G D}\right)}=f_{T} \cdot\left[1+\frac{R_{S}+R_{D}}{R_{D S}}+\frac{C_{G D}}{C_{G S}+C_{G D}} \cdot g_{m 0} \cdot\left(R_{S}+R_{D}\right)\right]
$$

where

$$
C_{c s}=\frac{C_{s s 0}}{\sqrt{1-V_{g s} / V_{b i}}}, \quad C_{G D}=\frac{C_{s s 0}}{\sqrt{1-V_{g d} / V_{b i}}}
$$

respectively. $C_{g s 0}$ is the zero-bias gate-source capacitance, whereas $V_{g d}=V_{G}-I_{D} R_{S}$ and $V_{g d}$ $=V_{G^{-}}\left(V_{D^{-}} I_{D} R_{D}\right)$ are the voltage-drop across the p-n junction on either side of the channel, respectively. From the above equations, we obtain

$$
f_{T 0}=f_{T} \cdot\left\lfloor 1+\frac{R_{S}+R_{b}}{R_{D S}}+\frac{g_{m 0} \cdot\left(R_{S}+R_{D}\right) \cdot \sqrt{V_{b i}-\left(V_{G}-I_{D} R_{S}\right)}}{\sqrt{V_{b i}-\left(V_{G}-I_{D} R_{S}\right)}+\sqrt{V_{b i}-\left(V_{G}-V_{D}+I_{D} R_{D}\right)}}\right\rfloor
$$

Since all the variables on the right hand side are directly measurable, this equation provides a simple method to extract the intrinsic $f_{T O}$ from parasitic-limited FETs. An $f_{T O}$ of $10 \mathrm{GHz}$ was calculated for GaN JFETs using DC measurement results.

In order to estimate the ultimate speed that can be achieved in our JFETs, a calculation was made to simulate the change in $g_{m}$ as $L_{g}$ decreases. Figure 2 shows the calculated $g_{m}$ vs. $L_{g}$ curves for various saturation velocity $\left(v_{s}\right)$. A $60 \%$ increase was obtained in $g_{m}$ as the $L_{g}$ reduced from 0.8 to $0.1 \mu \mathrm{m}$. By fitting the $g_{m}$ of the GaN JFETs into this plot, a $v_{s}$ of $6.6 \times 10^{6} \mathrm{~cm} / \mathrm{s}$ was obtained. Notice that the gate capacitance decreases linearly as the gate length shrinks. In addition, the electron velocity can exceed the $v_{s}$ in ultra-short gate length FETs due to velocity overshoot under high electrical field. Considering all these effects, an $f_{T}$ in excess of $50 \mathrm{GHz}$ could be expected in GaN JFETs upon further improving the device design and process techniques, and scaling down the gate length. Also shown in Figure 2 were the reported $g_{m}$ values of GaN MODFETs and MESFETs. The data are somewhat scattered, possibly due to different material quality for different research groups. The $g_{m}$ of GaN JFET is comparable to most of them, suggesting a reasonably good quality

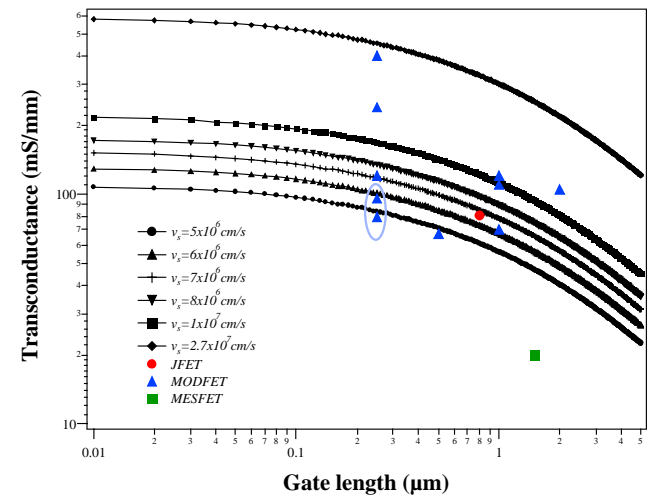

F99W4.9 
Figure 2: Simulation result of $g_{m}$ as a function of $L_{g}$ for various $v_{s}$ values. The transconductance of GaN JFETs and reported GaN MODFETs and MESFETs are also shown for comparison.

of JFET material.

In the above analysis, the effect of non-ideal ohmic contact to $\mathrm{p}-\mathrm{GaN}$ on the $g_{m}$ and $f_{T}$ has not been considered. For microwave operation, the large gate displacement current causes a significant voltage drop on the gate resistor, leading to a reduced $g_{m}$ and $f_{T}$. In addition, the extra capacitance at metal-p-GaN interface caused by the Schottky-type $\mathrm{Ni} / \mathrm{Au}$ to $\mathrm{p}-\mathrm{GaN}$ contact results in a further reduction in the $f_{T}$. In order to optimize the performance of GaN JFETs, a low resistance metal contact to $\mathrm{p}-\mathrm{GaN}$ with ideal ohmic I-V characteristic needs to be developed.

The DC performance of the GaN JFETs was also studied at elevated temperatures. The measurement was made on a hot plate in atmosphere. Figure 3 shows the plots of $I_{D}$ and $g_{m}$ as a function of $V_{G}$ at different temperatures. A continuous deterioration in $I_{D}$ and $g_{m}$ was observed with increasing temperatures up to $200^{\circ} \mathrm{C}$ where the device failed after being heated in the air for $\sim 2$ hours. As the temperature was increased from $25^{\circ} \mathrm{C}$ to $200^{\circ} \mathrm{C}, I_{D}$ dropped from $270 \mathrm{~mA} / \mathrm{mm}$ to $75 \mathrm{~mA} / \mathrm{mm}$ and $g_{m}$ was reduced from $48 \mathrm{mS} / \mathrm{mm}$ to $12 \mathrm{mS} / \mathrm{mm}$, respectively. This was mainly caused by the reduction in electron mobility at elevated temperatures due to enhanced polar optical phonon scattering. However, the $I_{D^{-}} V_{D}$ curve exhibited an excellent pinchoff at a gate bias of $-8 \mathrm{~V}$, indicating a negligible gate leakage current. The failure of the JFETs after high temperature measurement was caused by the degradation of $\mathrm{Ti} / \mathrm{Al}$ ohmic contact. An inspection of the devices after measurement showed a broken metal connection at the sidewall of the isolation mesa. The mesa profile was highly anisotropic, which resulted in a poor coverage of $\mathrm{Ti} / \mathrm{Al}$ metal over the sidewall. Hence, the oxidation of $\mathrm{Al}$ upon prolonged heating in the air caused an open circuit.
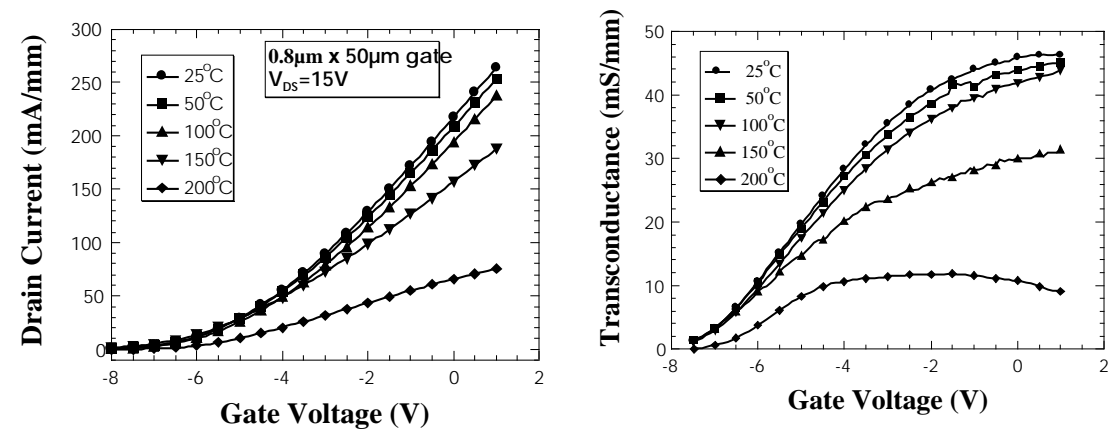

Figure 3: Drain current and transconductance as a function of gate voltage and measurement temperature.

\section{CONCLUSION}

In summary, JFETs were fabricated on an epitaxially grown GaN p-n junction. These devices exhibited excellent pinch-off and a breakdown voltage that agreed with theoretical predictions. An extrinsic transconductance of $48 \mathrm{mS} / \mathrm{mm}$ was achieved with a maximum $I_{D}$ of $270 \mathrm{~mA} / \mathrm{mm}$. Drain current collapse was observed in these devices after a high drain 
bias was applied due to partially depleted channel by the trapped electrons in the SI-GaN. The microwave measurement showed an $f_{T}$ of $6 \mathrm{GHz}$ and an $f_{\max }$ of $12 \mathrm{GHz}$. A simple method was developed to extract $f_{T O}$ from parasitic-limited FETs. An $f_{T O}$ of $10 \mathrm{GHz}$ was calculated for GaN JFETs using DC and rf results.

\section{ACKNOWLEDGEMENT}

This work was supported in part by the National Science Foundation CAREER Grant ECS-9501785. Sandia is a multiprogram laboratory operated by Sandia Corporation, a Lockheed Martin Company, for the United States Department of Energy under contract DE-ACO4-94AL85000.

\section{References}

1. V. A. Dmitriev, K. G. Irvine, C. H. Carter Jr., I. Kuznetsov, and E. V. Kalinina, Appl. Phys. Lett., 68, 229 (1996).

2. B. Gelmont, K. Kim, and M. S. Shur, J. Appl. Phys., 74, 1818 (1993).

3. S. N. Mohammad, A. Salvador, and H. Morkoç, Proc. IEEE 83, 1306 (1995).

4. S. C. Binari, W. Kruppa, H. B. Dietrich, G. Kelner, A. E. Wickenden, and J. A. Freitas JR, Solid-St. Electron., 41, 1549 (1997).

5. S. Yoshida and J. Suzuki, J. Appl. Phys., 84, 2940 (1998).

6. U. K. Mishra, Y. F. Wu, B. P. Keller, S. Keller, and S. P. Denbaars, IEEE Trans. Microwave Theory Tech., 46, 756 (1998).

7. M. A. Khan, Q. Chen, M. S. Shur, B. T. Dermott, J. A. Higgins, J. Burm, W. J. Schaff, and L. F. Eastman, Solid-St Electron., 41, 1555 (1997).

8. J. C. Zolper, R. J. Shul, A. G. Baca, R. G. Wilson, S. J. Pearton, R. A. Stall, Appl. Phys. Lett., 68, 2273 (1996).

9. C. H. Chen, S. Keller, G. Parish, R. Vetury, P. Kozodoy, E. L. Hu, S. P. Denbaars, U. K. Mishra, and Y. F. Wu, Appl. Phys. Lett., 73, 3147 (1998).

10. Y. F. Wu, B. P. Keller, D. Kapolnek, P. Kozodoy, S. P. Denbaars, and U. K. Mishra, Appl. Phys. Lett., 69, 1438 (1996).

11. Y. F. Wu, B. P. Keller, P. Fini, J. Pusl, M. Le, N. X. Nguyen, C. Nguyen, D. Widmen, S. Keller, S. P. Denbaars, and U. K. Mishra, Electron. Lett., 33, 1742 (1997).

12. L. F. Eastman, K. Chu, W. Schaff, M. Murphy, N. G. Weimann, and T. Eustis, MRS Internet J. Nitride Semicond. Res., 2, art. 17 (1997).

13. S. C. Binari, J. M. Redwing, G. Kelner, and W. Kruppa, Electron. Lett., 33, 242 (1997).

14. Y. F. Wu, B. P. Keller, S. Keller, D. Kapolnek, P. Kozodoy, S. P. Denbarrs, and U. K. Mishra, Solid-St. Electron., 41, 1569 (1997).

15. A. Osinski, S. Gangopadhyay, B. W. Lim, M. Z. Anwar, M. A. Khan, D. V. Kuksenkov, and H. Temkin, Appl. Phys. Lett., 72, 742 (1998).

16. J. M. Van Hove, R. Hichman, J. J. Klaassen, P. P. Chow, and P. P. Ruden, Appl. Phys. Lett., 70, 2282 (1997).

17. C. Pernot, A. Hirano, H. Amano, and I. Akasaki, Jpn. J. Appl. Phys., 37, L1202 (1998). 
18. L. Zhang, L. F. Lester, A. G. Baca, R. J. Shul, P. C. Chang, C. G. Willison, U. K. Mishra, S. P. Denbaars, and J. C. Zolper, IEEE Electron Device Lett., accepted.

19. O. Aktas, Z. F. Fan, A. Botchkarev, S. N. Mohammad, M. Roth, T. Jenkins, L. Kehias, and H. Morkoç, IEEE Electron Device Lett., 18, 293 (1997).

20. Y. F. Wu, B. P. Keller, S. Keller, D. Kapolnek, S. P. Denbarrs, and U. K. Mishra, IEEE Electron Device Lett., 17, 455 (1996).

21. P. J. Tasker and B. Hughes, IEEE Electron Device Lett., 10, 291 (1989). 